\title{
Joint symptoms associated with anastrozole and letrozole in patients with breast cancer: a retrospective comparative study
}

\author{
Yoshihito Morimoto $^{1 *}$ D, Shuhei Sarumaru², Yuko Oshima' ${ }^{1}$, Chiho Tsuruta ${ }^{1}$ and Kazuhiro Watanabe ${ }^{1}$
}

\begin{abstract}
Background: Joint symptoms are a common side effect of aromatase inhibitors. However, it is not known if the risk of these symptoms varies between the members of this drug class. The aim of this study was to compare the frequency of joint symptoms associated with anastrozole and that associated with letrozole.

Methods: We retrospectively reviewed patients with breast cancer who were treated with anastrozole or letrozole at Tsukiji Breast Clinic, Japan, between April 2008 and July 2014. Joint symptoms were deemed to include both joint pain and painless joint symptoms. The time to onset of joint symptoms in the anastrozole group was compared with that in the letrozole group using Kaplan-Meier curves and the log-rank test.

Results: Of 141 patients identified to have received aromatase inhibitors, 70 had been treated with anastrozole and 71 with letrozole. Joint symptoms occurred in $60.3 \%$ of the 141 patients $(60.0 \%$ in the anastrozole group and $60.6 \%$ in the letrozole group; $p=1$ ). Median time to appearance of joint symptoms was 583 days, with no significant difference between the anastrozole and letrozole groups ( $p=0.962)$. There was no significant difference in time to onset of joint pain ( $p=0.139)$; however, time to onset of painless joint symptoms was significantly shorter in the anastrozole group $(p=0.022)$. The sites at which joint symptoms occurred were similar in the two groups.
\end{abstract}

Conclusions: The results of this study indicate that there is no difference in the pattern of occurrence of joint symptoms caused by anastrozole and those caused by letrozole.

Trial registration: Trial registration was not required for this study because of its retrospective nature and lack of intervention.

Keywords: Anastrozole, Aromatase inhibitors, Breast cancer, Joint symptoms, Letrozole

\section{Background}

Aromatase inhibitors are frequently used to treat postmenopausal women with hormone receptor-positive breast cancer. Anastrozole has demonstrable efficacy compared with tamoxifen as adjuvant therapy for postmenopausal women with this disease [1]. The Breast International Group (BIG) 1-98 study also reported that letrozole is effective and safe when used as adjuvant

\footnotetext{
* Correspondence: y-morimoto@ac.shoyaku.ac.jp

${ }^{1}$ Education and Research Center for Clinical Pharmacy, Showa

Pharmaceutical University, 3-3165 Higashi-Tamagawagakuen, Machida, Tokyo 194-8543, Japan

Full list of author information is available at the end of the article
}

monotherapy [2]. Furthermore, a meta-analysis showed that aromatase inhibitors improve overall survival in postmenopausal women with hormone receptor-positive advanced breast cancer [3]. Therefore, aromatase inhibitors are recommended as primary endocrine therapy for these women. However, these agents are associated with a high frequency of joint symptoms. The ATAC trial showed that the frequency of arthralgia attributable to anastrozole was $35.6 \%$ [4]. In the BIG 1-98 study, letrozole caused arthralgia in $20.0 \%$ of patients [5]. Furthermore, in a Japanese study, joint pain and stiffness occurred in 61.6 and $59.2 \%$, respectively, of women treated with anastrozole [6]. However, although it is 
known that aromatase inhibitors are associated with a high rate of joint symptoms, comparative data for the agents in this class are limited. The incidence of joint symptoms may differ between anastrozole and letrozole.

The aim of this study was to compare the frequency, time to onset, and sites of joint symptoms associated with anastrozole and letrozole in patients with breast cancer.

\section{Methods}

The study protocol was approved by the research ethics committee of Showa Pharmaceutical University (approval numbers 2011-17 and 2014-3) and conducted in accordance with the Declaration of Helsinki. The need for informed consent was waived in view of the retrospective and observational nature of the study. Patients who had been prescribed anastrozole or letrozole at the Tsukiji Breast Clinic between April 2008 and July 2014 were identified. Patients who made fewer than 10 visits to the clinic and those who had joint symptoms prior to administration of aromatase inhibitors were excluded, as were patients who had received combination chemotherapy.

\section{Evaluation}

Data on patient characteristics and occurrence and site of joint symptoms were collected. Joint symptoms were defined as either joint pain or painless joint symptoms, such as joint stiffness and decreased joint motion. Joint symptoms were defined as joint pain or painless joint symptoms that occurred on at least one occasion. All the joint symptoms associated with aromatase inhibitors were evaluated during interviews conducted by the same doctor.

\section{Statistical analysis}

The Mann-Whitney $U$ test and Fisher's exact test were used to identify any differences in patient characteristics between the anastrozole and letrozole groups. The frequency of joint symptoms was compared between the two treatment groups using Fisher's exact test. Time to onset of joint symptoms was compared between the two groups using Kaplan-Meier curves and the log-rank test. The starting point of the Kaplan-Meier curves was the day on which an aromatase inhibitor was started. The statistical analysis was performed using EZR version 1.35 software ( $\mathrm{R}$ Foundation for Statistical Computing, Vienna, Austria) [7]. A $p$-value $<0.05$ was considered to be statistically significant.

\section{Results}

\section{Patient characteristics}

Data were obtained for 141 patients (anastrozole group, $n=70$; letrozole group, $n=71$ ). Patient characteristics are shown in Table 1 . Significantly more women had a
Table 1 Patient characteristics

\begin{tabular}{|c|c|c|c|}
\hline & Anastrozole & Letrozole & $p$-value \\
\hline Patients (n) & 70 & 71 & \\
\hline Median age, years (range) & $64(51-84)$ & $64(49-88)$ & $0.579^{a}$ \\
\hline \multicolumn{4}{|l|}{ Stage } \\
\hline 0 & 1 & 0 & \\
\hline । & 20 & 15 & \\
\hline$\| \mathrm{A}$ & 25 & 21 & \\
\hline$\| \mathrm{B}$ & 6 & 14 & \\
\hline$\| I I A$ & 5 & 8 & \\
\hline$\| I B$ & 5 & 4 & \\
\hline$\| I I C$ & 2 & 3 & \\
\hline IV & 1 & 1 & \\
\hline Recurrence & 4 & 4 & \\
\hline Unknown & 1 & 1 & \\
\hline \multicolumn{4}{|l|}{ History of hormone therapy } \\
\hline Yes & 10 & 24 & $<0.01^{\mathrm{b}}$ \\
\hline No & 60 & 47 & \\
\hline \multicolumn{4}{|l|}{ History of chemotherapy } \\
\hline Yes & 27 & 41 & $0.029^{b}$ \\
\hline No & 43 & 30 & \\
\hline
\end{tabular}

a Mann-Whitney $U$ test

${ }^{b}$ Fisher's exact test

history of receiving hormone therapy in the letrozole group than in the anastrozole group $(p<0.01)$. All patients with a history of hormone therapy, except for one in the letrozole group, had received tamoxifen. Significantly more women in the letrozole group had a history of chemotherapy than in the anastrozole group $(p=0.029)$.

\section{Frequency of joint symptoms}

Table 2 shows the frequency of joint symptoms. The overall frequency of joint symptoms was $60.3 \%$ (60.0\% in the anastrozole group versus $60.6 \%$ in the letrozole group; $p=1$ ). Painless joint symptoms occurred significantly more often in the anastrozole group than in the letrozole group ( $41.4 \%$ vs. $23.9 \%$; $p=0.032)$. However, there was no significant difference in occurrence of joint pain between the groups $(p=0.239)$.

\section{Time to onset of joint symptoms}

Figure 1a shows Kaplan-Meier curves for the overall rate of joint symptoms. Median time until occurrence of joint symptoms was 583 days. Figure 1b shows Kaplan-Meier curves for the rate of development of joint symptoms in the anastrozole and letrozole groups. Median time until occurrence of joint symptoms was not significantly different between the two 
Table 2 Frequency of joint symptoms

\begin{tabular}{lllll}
\hline & Total & Anastrozole & Letrozole & $p$-value \\
\hline Patients ( $\mathrm{n})$ & 141 & 70 & 71 & \\
Joint symptoms (\%) & $85(60.3)$ & $42(60.0)$ & $43(60.6)$ & $1^{\mathrm{a}}$ \\
$\quad$ Joint pain (\%) & $66(46.8)$ & $29(41.4)$ & $37(52.1)$ & $0.239^{\mathrm{a}}$ \\
$\begin{array}{l}\text { Painless joint symptoms } \\
(\%)\end{array}$ & $46(32.6)$ & $29(41.4)$ & $17(23.9)$ & $0.032^{\mathrm{a}}$ \\
\hline
\end{tabular}

${ }^{\mathrm{a}}$ Fisher's exact test

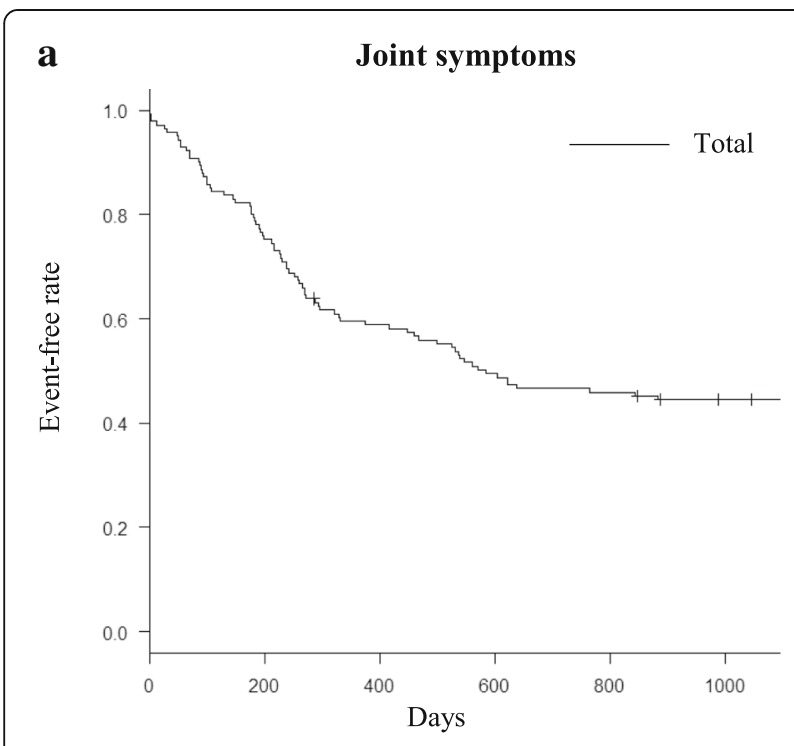

b

Joint symptoms

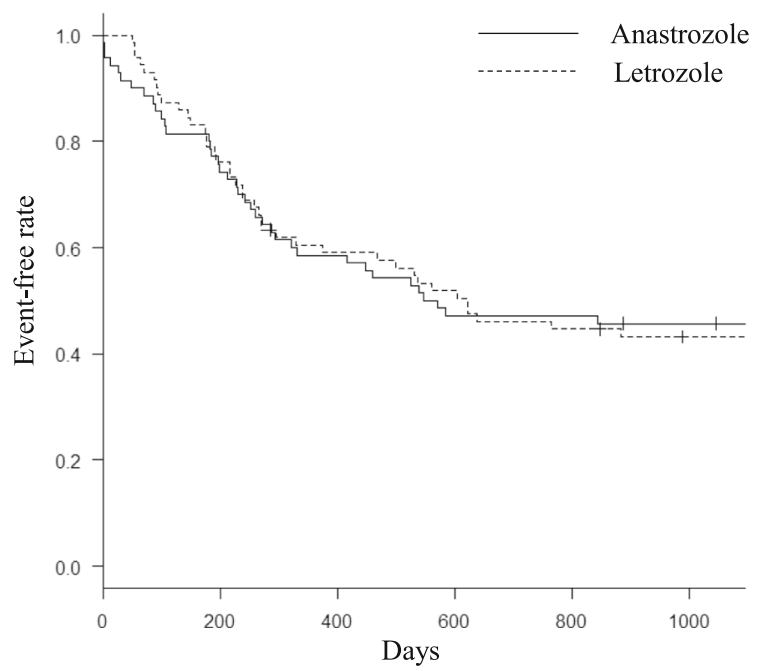

Fig. 1 Event-free curves for joint symptoms. a Combined data and (b) data for anastrozole vs. letrozole. There was no statistically significant difference in time to onset of joint symptoms between the anastrozole and letrozole groups ( $p=0.962)$ groups (558.5 days in the anastrozole group and 621 days in the letrozole group; $p=0.962$ ). Figure $2 \mathrm{a}$, b shows the respective Kaplan-Meier curves for the rates of joint pain and painless joint symptoms. Time to onset of painless joint symptoms was significantly shorter in the anastrozole group than in the letrozole group $(p=0.022)$. However, there was no significant difference in time to onset of joint pain between the two groups $(p=0.139)$.

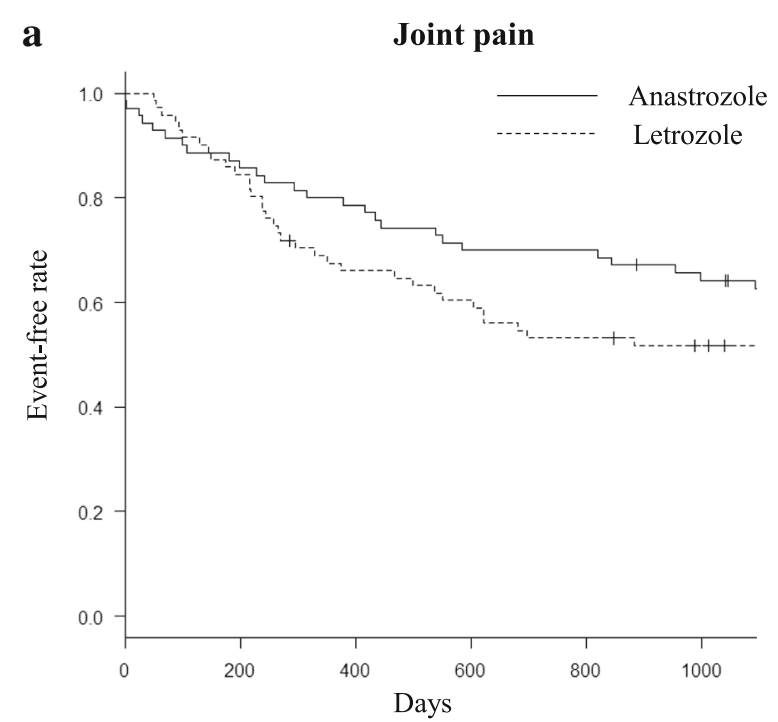

b

Painless joint symptoms

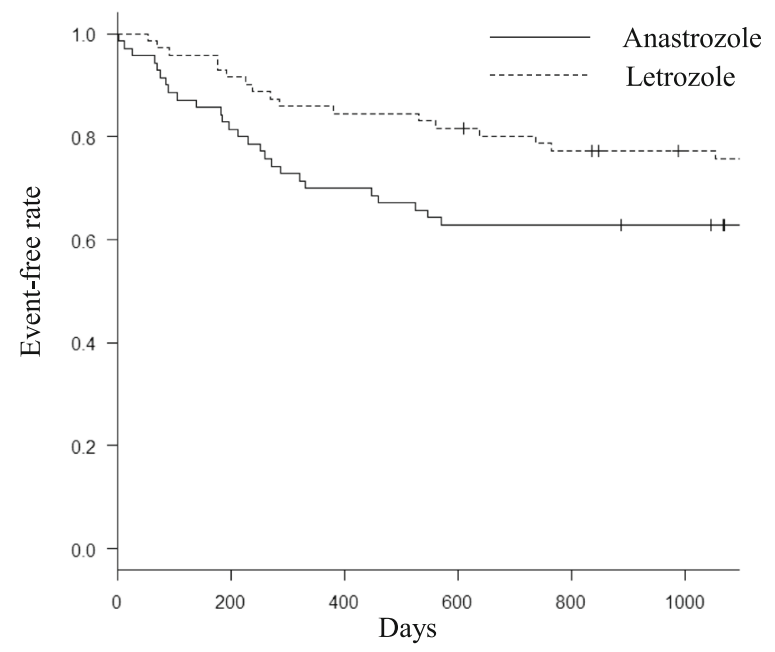

Fig. 2 Event-free curves for (a) joint pain and (b) painless joint symptoms for anastrozole vs. letrozole. There was no statistically significant difference in occurrence of joint pain $(\mathbf{a}, p=0.139$, logrank test). However, the time to onset of painless joint symptoms was significantly shorter in the anastrozole group than in the letrozole group (b, $p=0.022$, log-rank test) 


\section{Sites of joint symptoms}

Table 3 shows the sites of joint pain and painless joint symptoms in the anastrozole and letrozole groups. Joint pain occurred at various sites in both groups, although the data were incomplete for the letrozole group. In both groups, the most frequently reported site of painless joint symptoms was the wrist/hand.

\section{Discussion}

In this study, there was no difference in the frequency or time to onset of joint symptoms between women who received anastrozole and those who received letrozole. Aromatase inhibitor-related joint symptoms are known to be common. Yagata et al. reported a high rate of joint pain and stiffness associated with anastrozole [6], and a prospective multicenter cohort study in Japan reported a $71.8 \%$ rate of new or worsening joint symptoms in women receiving anastrozole [8]. Furthermore, Mao et al. reported rates of arthralgia associated with anastrozole, letrozole, and exemestane of 45,49 , and $48 \%$, respectively, with no significant difference in the rates between treatment groups [9]. We also found a high rate of joint symptoms with anastrozole and letrozole (60.0 and 60.6\%, respectively) and no significant difference between the groups. Ours is the first study to compare the rates and times to onset of joint symptoms between anastrozole and

Table 3 Site of joint symptoms (joint pain and painless joint symptoms)

\begin{tabular}{lll}
\hline & Anastrozole & Letrozole \\
\hline Joint pain & 5 & 5 \\
Wrist/Hand & 0 & 0 \\
Elbow & 5 & 8 \\
Shoulder & 0 & 1 \\
Back & 3 & 2 \\
Hip & 9 & 4 \\
Knee & 2 & 3 \\
Feet & 4 & 0 \\
Total body & 1 & 14 \\
Unknown & & \\
Painless joint symptoms & 15 & 15 \\
Wrist/Hand & 1 & 0 \\
Elbow & 1 & 0 \\
Shoulder & 2 & 1 \\
Back & 0 & 0 \\
Hip & 3 & 0 \\
Knee & 0 & 3 \\
Feet & 1 & 1 \\
Total body & 3 & 0 \\
Unknown & & \\
\hline
\end{tabular}

letrozole using Kaplan-Meier curves. There was no significant difference in time to onset of joint symptoms between the two groups; in most cases, joint symptoms occurred after more than 6 months of receiving aromatase inhibitor therapy, although symptoms did appear during the first 3 months of treatment in some cases. This finding suggests that aromatase inhibitor-related joint symptoms tend to develop late in treatment and should be watched for at this time. However, there have been reports of joint symptoms developing within the first 6 months of treatment $[8,9]$. This discrepancy could be attributable to differences in background characteristics between the patients in the previous studies and those in our present study, and the fact that data were only collected for 1 year in the previous studies. Kanematsu et al. also reported that the onset of aromatase inhibitor-related arthralgia was sometimes delayed [10]. In the anastrozole group, the main sites of joint pain and painless joint symptoms were the knee and hand, respectively, as found in a previous study [6].

Anastrozole and letrozole improve disease-free survival in patients with breast cancer to a similar extent and have replaced tamoxifen as the adjuvant standard of care. However, anastrozole and letrozole differ in their structure, and both in vitro and animal models have shown differences in their mechanism and potency of estrogen suppression [11]. Geisler et al. identified letrozole to be a more potent suppressor of total body aromatization and plasma estrogen levels than anastrozole in postmenopausal women with metastatic breast cancer [12]. A head-to-head comparison (the Femara Versus Anastrozole Clinical Evaluation trial) is now under way to verify this difference in clinical effect [11]. In another study, there was no significant difference in quality of life scores between anastrozole and letrozole [13], but further evidence comparing these 2 drugs is awaited.

Although the exact mechanism of the joint symptoms associated with the aromatase inhibitors is still unclear, an aromatase inhibitor-induced decrease in estrogen levels might play an important role in the pathogenesis of these joint symptoms [14]. In our study, the rate of painless joint symptoms was significantly higher in the anastrozole group than in the letrozole group and the time to onset of these symptoms was significantly shorter in the anastrozole group than in the letrozole group. Joint pain tended to develop earlier in the letrozole group than in the anastrozole group, but the difference was not statistically significant. The reasons for these differences are unknown, but may lie in differences in the extent of suppression of total aromatization and plasma estrogen levels between anastrozole and letrozole. A prospective trial would be needed to confirm if such a difference exists. 
Patient tolerability and compliance with aromatase inhibitor therapy is a very important issue because of the extended duration of treatment involved. In addition to causing joint symptoms, the side effects of aromatase inhibitors include hot flashes, osteoporosis, fractures, hypercholesterolemia, and cardiovascular events [11]. Joint symptoms adversely impact health-related quality of life in many patients and reduce compliance. We hope that our findings will help in selection of appropriate adjuvant therapy for women with breast cancer.

The present study has several limitations. First, it had a retrospective, single-center design and a small sample size. Furthermore, a significantly greater proportion of women in the letrozole group had a history of hormone therapy when compared with the anastrozole group. Previous hormone therapy is thought to be associated with joint symptoms $[15,16]$. Similarly, a significantly greater number of women had a history of chemotherapy in the letrozole group than in the anastrozole group, so the patient background characteristics were not comparable. Taxane chemotherapy has been reported to contribute to expression of joint symptoms with aromatase inhibitors $[14,15]$. Second, patients with breast cancer may also have symptoms of menopause, which can be difficult to distinguish from the side effects of aromatase inhibitors. We excluded patients with joint symptoms before administration of an aromatase inhibitor to eliminate this potential source of confounding as far as possible.

\section{Conclusion}

No difference was observed in the rate of joint symptoms associated with administration of anastrozole and letrozole.

\section{Acknowledgments}

Not applicable

\section{Funding}

Not applicable

\section{Availability of data and materials}

The data sets generated and/or analyzed in this study are not publicly available for privacy reasons, but are available from the corresponding author on reasonable request.

\section{Authors' contributions}

YM analyzed the data and wrote the manuscript. KW designed and supervised the research. SS interviewed patients and supervised the research. YO and CT collected the data. All authors read and approved the final manuscript.

\section{Ethics approval and consent to participate}

The study protocol was approved by the research ethics committee of Showa Pharmaceutical University (approval numbers 2011-17 and 2014-3) and conducted in accordance with the Declaration of Helsinki. This study was retrospective and noninterventional, so informed consent was not deemed necessary.
Consent for publication

Not applicable

\section{Competing interests}

The authors declare that they have no competing interests.

\section{Publisher's Note}

Springer Nature remains neutral with regard to jurisdictional claims in published maps and institutional affiliations.

\section{Author details}

${ }^{1}$ Education and Research Center for Clinical Pharmacy, Showa Pharmaceutical University, 3-3165 Higashi-Tamagawagakuen, Machida, Tokyo 194-8543, Japan. ${ }^{2}$ Breast Clinic Tsukiji, Kaken Tsukiji Building 4F, 11-6 Akashi-cho, Chuo-ku, Tokyo 104-0044, Japan.

Received: 5 September 2017 Accepted: 26 October 2017

Published online: 07 November 2017

\section{References}

1. Cuzick J, Sestak I, Baum M, Buzdar A, Howell A, Dowsett M, et al. ATAC/ LATTE investigators. Effect of anastrozole and tamoxifen as adjuvant treatment for early-stage breast cancer: 10-year analysis of the ATAC trial. Lancet Oncol. 2010, 11:1135-41.

2. BIG 1-98 Collaborative Group, Mouridsen H, Giobbie-Hurder A, Goldhirsch A, Thürlimann B, Paridaens R, et al. Letrozole therapy alone or in sequence with tamoxifen in women with breast cancer. N Engl J Med. 2009:361:766-76.

3. Mauri D, Pavlidis N, Polyzos NP, loannidis JP. Survival with aromatase inhibitors and inactivators versus standard hormonal therapy in advanced breast cancer: meta-analysis. J Natl Cancer Inst. 2006;98:1285-91.

4. Howell A, Cuzick J, Baum M, Buzdar A, Dowsett M, Forbes JF, et al. ATAC Trialists' Group. Results of the ATAC (Arimidex, Tamoxifen, alone or in combination) trial after completion of 5 years' adjuvant treatment for breast cancer. Lancet. 2005:365:60-2.

5. Coates AS, Keshaviah A, Thürlimann B, Mouridsen H, Mauriac L, Forbes JF, et al. Five years of letrozole compared with tamoxifen as initial adjuvant therapy for postmenopausal women with endocrine-responsive early breast cancer: update of study BIG 1-98. J Clin Oncol. 2007;25:486-92.

6. Yagata H, Ohtsu H, Komoike Y, Saji S, Takei H, Nakamura T, et al. Joint symptoms and health-related quality of life in postmenopausal women with breast cancer who completed 5 years of anastrozole. Support Care Cancer. 2016;24:683-9.

7. Kanda Y. Investigation of the freely available easy-to-use software 'EZR' for medical statistics. Bone Marrow Transplant. 2013;48:452-8.

8. Egawa C, Hirokaga K, Takao S, Yamagami K, Miyashita M, Baba M, et al. Risk factors for joint symptoms in postmenopausal Japanese breast cancer patients treated with anastrozole: a prospective multicenter cohort study of patient-reported outcomes. Int J Clin Oncol. 2016;21:262-9.

9. Mao JJ, Stricker C, Bruner D, Xie S, Bowman MA, Farrar JT, et al. Patterns and risk factors associated with aromatase inhibitor-related arthralgia among breast cancer survivors. Cancer. 2009;115:3631-9.

10. Kanematsu M, Morimoto M, Honda J, Nagao T, Nakagawa M, Takahashi M, et al. The time since last menstrual period is important as a clinical predictor for non-steroidal aromatase inhibitor-related arthralgia. BMC Cancer. 2011;11:436.

11. Geisler J. Differences between the non-steroidal aromatase inhibitors anastrozole and letrozole-of clinical importance? Br J Cancer. 2011;104:1059-66.

12. Geisler J, Haynes B, Anker G, Dowsett M, Lønning PE. Influence of letrozole and anastrozole on total body aromatization and plasma estrogen levels in postmenopausal breast cancer patients evaluated in a randomized, crossover study. J Clin Oncol. 2002;20:751-7.

13. Dixon JM, Renshaw L, Langridge $C$, Young $O E$, McHugh $M$, Williams $L$, et al. Anastrozole and letrozole: an investigation and comparison of quality of life and tolerability. Breast Cancer Res Treat. 2011;125:741-9.

14. Okishiro M, Taguchi T, Kim SJ, Tanji Y, Shimazu K, Tamaki Y, et al. Incidence of joint symptoms and bone fractures in Japanese postmenopausal breast cancer patients treated with adjuvant anastrozole. J Cancer Res Clin Oncol. 2009;135:823-7. 
15. Crew KD, Greenlee H, Capodice J, Raptis G, Brafman L, Fuentes D, et al. Prevalence of joint symptoms in postmenopausal women taking aromatase inhibitors for early-stage breast cancer. J Clin Oncol. 2007;25:3877-83.

16. Sestak I, Cuzick J, Sapunar F, Eastell R, Forbes JF, Bianco AR, et al. Risk factors for joint symptoms in patients enrolled in the ATAC trial: a retrospective, exploratory analysis. Lancet Oncol. 2008;9:866-72.

Submit your next manuscript to BioMed Central and we will help you at every step:

- We accept pre-submission inquiries

- Our selector tool helps you to find the most relevant journal

- We provide round the clock customer support

- Convenient online submission

- Thorough peer review

- Inclusion in PubMed and all major indexing services

- Maximum visibility for your research

Submit your manuscript at www.biomedcentral.com/submit 